\title{
Analysis of IS-95 CDMA Voice Privacy
}

\author{
Muxiang Zhang ${ }^{1}$, Christopher Carroll ${ }^{1}$, and Agnes Chan ${ }^{2}$ \\ 1 GTE Laboratories Inc., 40 Sylvan Road LA0MS59, Waltham, MA 02451 \\ \{mzhang, ccarroll\}@gte.com \\ ${ }^{2}$ College of Computer Science, Northeastern University, Boston, MA 02115 \\ ahchan@ccs.neu.edu
}

\begin{abstract}
The voice privacy of IS-95 CDMA cellular system is analyzed in this paper. By exploiting information redundancy on the downlink traffic channel, it is shown that an eavesdropper can recover the voice privacy mask after eavesdropping the transmission on the downlink traffic channel for about one second. Thus, IS-95 CDMA voice privacy is vulnerable under ciphertext-only attacks.
\end{abstract}

\section{Introduction}

IS-95 Code Division Multiple Access (CDMA) is an interim industry standard 3 . for cellular communication systems. In IS-95 CDMA, a pseudo-random pattern, which is a high bit-rate binary sequence known as long code sequence, is added to the low bit-rate voice signal. Adding a high bit-rate noise-like signal to a voice signal makes the voice signal more robust and less susceptible to interference. It enables low-power transmission to take place, resulting in cheaper mobile stations with long-lasting battery life. The long code sequence, which is only known to the designated receiver, is also expected to provide certain level of privacy to the voice signal. To decode the voice signal, the eavesdropper has to recover the long code sequence from the intercepted signal. The long code sequence is generated by the long code generator as shown in Figure 3. The long code generator consists of a 42-bit number called long code mask and a 42-bit linear feedback shift register (LFSR) specified by the following characteristic polynomial:

$$
\begin{aligned}
& x^{42}+x^{35}+x^{33}+x^{31}+x^{27}+x^{26}+x^{25}+x^{22}+x^{21}+x^{19} \\
& +x^{18}+x^{17}+x^{16}+x^{10}+x^{7}+x^{6}+x^{5}+x^{3}+x^{2}+x+1
\end{aligned}
$$

The inner product of the LFSR state and the long code mask produces the long code sequence.

Voice privacy of IS-95 CDMA is provided by means of the long code mask. The long code mask is not transmitted through any channel, it is constructed by the base station and the mobile station. To recover the long code sequence, the eavesdropper may exhaustively search the 42-bit long code mask, with a time complexity of $O\left(2^{42}\right)$. This attack is viable but is hard to implement in real time. Alternatively, it can be shown that the long code sequence can also 


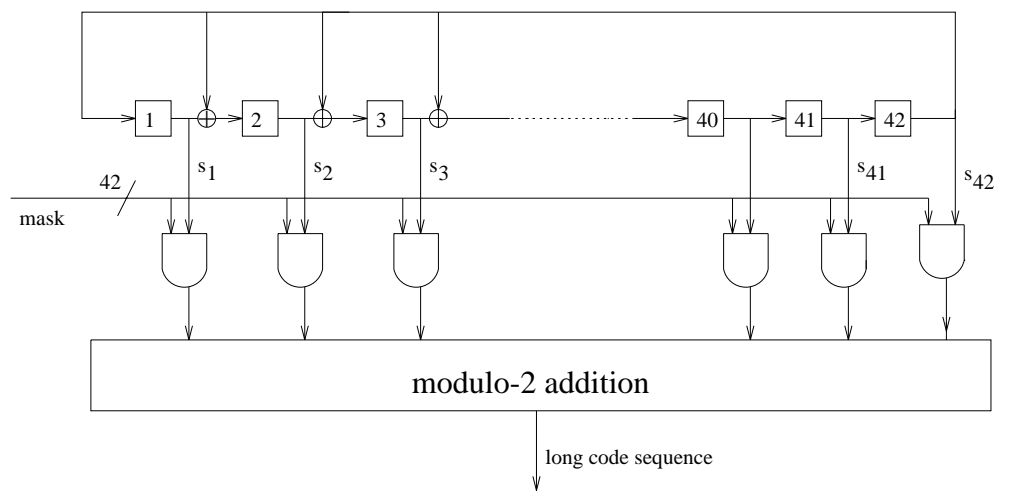

Fig. 1. The long code generator

be recovered if the eavesdropper can obtain 42 bits of plaintext-ciphertext pairs. As there are many mobile stations transmitting simultaneously on the traffic channel and each mobile station only transmits approximately 3 minutes on the average, it is rather difficult to obtain 42 bits of the plaintext message. In this paper, we investigate the security of IS-95 CDMA voice privacy under ciphertext-only attacks. By exploiting information redundancy introduced by channel coding, it is shown that the eavesdropper can recover the voice privacy mask after eavesdropping the transmission on the traffic channel for about one second. This paper is organized as follows. Section 2 gives an equivalent long code generator which can simplify the analysis of CDMA voice privacy. Section 3 describes two kinds of ciphertext-only attacks and Section 4 presents some concluding remarks.

\section{An Equivalent Long Code Generator}

Let $\left(m_{1}, m_{2}, \ldots m_{42}\right)$ denote the 42 -bit mask and $\left(s_{1}(k), s_{2}(k), \ldots, s_{42}(k)\right)$ denote the state of the LFSR at time instant $k$. Then the long code sequence $c(k)$ at time instant $k$ can be represented as

$$
c(k)=m_{1} s_{1}(k)+m_{2} s_{2}(k)+\cdots+m_{42} s_{42}(k),
$$

where the addition is the modulo-2 addition. Since $s_{1}(k), s_{2}(k), \cdots, s_{42}(k)$ are the outputs of the 42 stages of the LFSR, they are the same sequence but only differ in the phase. So, for any $i, 1 \leq i \leq 42, s_{i}(k)$ satisfies the following linear recurrence equation,

$$
s_{i}(k)=a_{1} s_{i}(k-1)+a_{2} s_{i}(k-2)+\cdots+a_{42} s_{i}(k-42),
$$

where $a_{i}$ is the coefficient of $x^{i}$ in the characteristic polynomial of the LFSR. Substituting (2) into (11), we have 


$$
\begin{aligned}
c(k) & =\sum_{i=1}^{42} m_{i} s_{i}(k) \\
& =\sum_{i=1}^{42} m_{i}\left(\sum_{j=1}^{42} a_{j} s_{i}(k-j)\right) \\
& =\sum_{i=1}^{42} a_{i}\left(\sum_{j=1}^{42} m_{j} s_{j}(k-i)\right) .
\end{aligned}
$$

By equation (1),

$$
\sum_{j=1}^{42} m_{j} s_{j}(k-i)=c(k-i) .
$$

Hence, it follows that

$$
c(k)=a_{1} c(k-1)+a_{2} c(k-2)+\cdots+a_{42} c(k-42),
$$

which means that the long code sequence is also generated by the same LFSR. Although every mobile station uses a different long code mask, their long code sequences are the same except for the different phases. The long code mask only affects the phase of the long code sequence.

Let $C(k)=(c(k), c(k-1), \cdots, c(k-41))$ and $C(k+1)=(c(k+1), c(k), \cdots$, $c(k-42))$ be two consecutive states of the LFSR. By equation (3), we have

$$
\left[\begin{array}{c}
c(k+1) \\
c(k) \\
\vdots \\
\vdots \\
c(k-42)
\end{array}\right]=\left[\begin{array}{ccccccc}
a_{1} & a_{2} & a_{3} & \cdots & a_{40} & a_{41} & a_{42} \\
1 & 0 & 0 & & 0 & 0 & 0 \\
0 & 1 & 0 & & 0 & 0 & 0 \\
0 & 0 & 1 & & 0 & 0 & 0 \\
\vdots & \vdots & \vdots & & \vdots & \vdots & \vdots \\
0 & 0 & 0 & \cdots & 1 & 0 & 0 \\
0 & 0 & 0 & \cdots & 0 & 1 & 0
\end{array}\right]\left[\begin{array}{c}
c(k) \\
c(k-1) \\
\vdots \\
c(k-41)
\end{array}\right] .
$$

Let $A^{t}$ denote the $42 \times 42$ matrix of the above equation and $a^{t}$ denote the vector $\left(a_{1}, a_{2}, \cdots, a_{42}\right)$, where $A^{t}$ is the transpose of $A$. Then for any $n>k$,

$$
C(n)=C(k) A^{n-k} .
$$

By equation (41), it follows that

$$
c(n)=C(k) A^{n-k-1} a .
$$

Equation (15) indicates that the state $C(k)$ of the LFSR can be calculated if any 42 bits of the long code sequence are known. Since the LFSR generating the long code sequence is publicly known, the coefficients $a_{i}, 1 \leq i \leq 42$ are available to the eavesdropper. Thus, the long code sequence can be generated if the eavesdropper can recover 42 bits of the long code sequence. 


\section{Attacks on CDMA Voice Privacy}

Before the analysis of IS-95 CDMA voice privacy, let us give a simple description of CDMA channels. The CDMA channels consists of traffic channels and control channels. The traffic channels carry user information while the control channels carry signalling information. The traffic channels can be further divided into downlink and uplink traffic channels.

\subsection{CDMA Traffic Channels}

The downlink channel carries information from the base station to the mobile station. On the downlink traffic channel, the vocoder accepts the voice signal and produces a compressed data stream. IS-95 CDMA specifies a variable-rate vocoder operating at full, $\frac{1}{2}, \frac{1}{4}$, and $\frac{1}{8}$ rates. The rate is determined according to the power level of the background noise. There are currently two types of vocoders: the one operating in a $9.6-\mathrm{kbps}$, and the other operating in a 14.4kbps, referred to as rate set 1 and rate set 2 , respectively. Rate set 1 contains four elements: 9.6, 4.8, 2.4, and $1.2 \mathrm{kbps}$. Rate set 2 also contains four elements: $14.4,7.2,3.6$, and $1.8 \mathrm{kbps}$. The mobile station has to support rate set 1 , while rate set 2 is optional.

The data stream from the vocoder is structured in 20 -ms frames. The full rate of rate set 1 vocoder is $8.6 \mathrm{kbps}$, which generates 172 bits every $20 \mathrm{~ms}$. The frame quality indicator, which is cyclic redundancy checking (CRC) digits derived from the 172 information bits, is added to the 172 bits along with an 8-bit tail (set to $0)$. The $9.6 \mathrm{kbps}$ frame is a result of 192 bits $(172+12+8)$ transmitted every 20 $\mathrm{ms}$. The 4.8-kbps frame has the same structure, while 2.4- and 1.2-kbps frames do not have frame quality indicator fields since most of the information sent in these frames is background noise. Rate set 2 frames have similar structures as rate set 1 . The 20 -ms data frames are encoded, interleaved, scrambled, spread, and modulated before they are sent onto the air interface. Figure 2 shows the different functions which act on rate set 1 data frames.

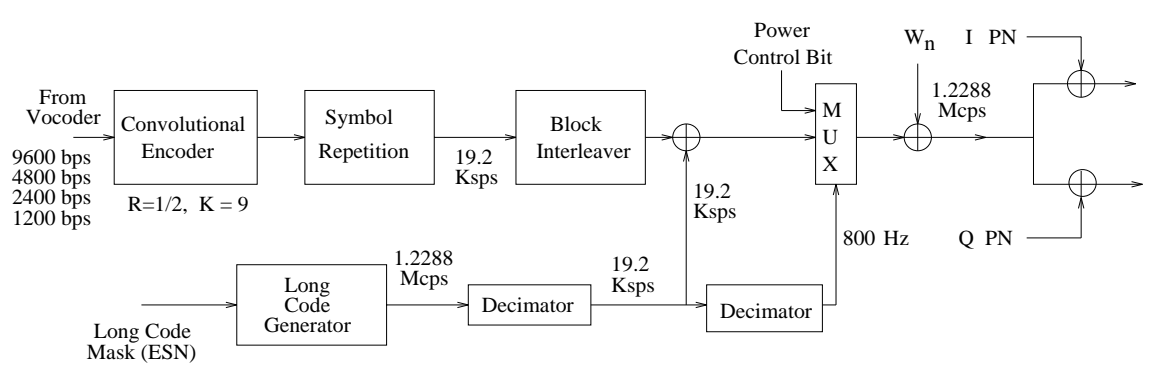

Fig. 2. Rate set 1 downlink traffic channel generation

The convolutional encoder provides error-correction capability to the downlink traffic channels. The convolutional encoder replace each single input bit with 
two bits (called code symbols). The symbol repeater repeats the code symbols produced by the convolutional encoder as necessary to result in an output stream with the fixed rate $19.2 \mathrm{ksps}$ (kilo-symbols per second). For example, to achieve this, it does not repeat anything if the input rate is $19.2 \mathrm{ksps}$. It repeats each symbol twice if the input rate is $9.6 \mathrm{ksps}$, it repeats each symbol 4 times if the input rate is $4.8 \mathrm{ksps}$. The block Interleaver shuffles the code symbols in each data frame. Data scrambling is provided through the long code generator. A power control subchannel is continuously transmitted on the downlink traffic channel. It is used to control the mobile station's power on the uplink. This subchannel transmits at a rate of one bit every $1.25 \mathrm{~ms}$ (i.e., $800 \mathrm{bps}$ ). The power control bit which is two symbols long replaces two consecutive symbols on the downlink traffic channel. The 19.2 ksps data stream, which has been punctured with the power control bits of the power control subchannel, is spread by a Walsh code. Following the Walsh code spreading, the data is modulated for transmission.

Like the downlink traffic channel, the uplink traffic channel also supports two rate sets, depending on the type of vocoder used. Figure 3 shows the overall structure of the uplink traffic channel for rate set 1 .

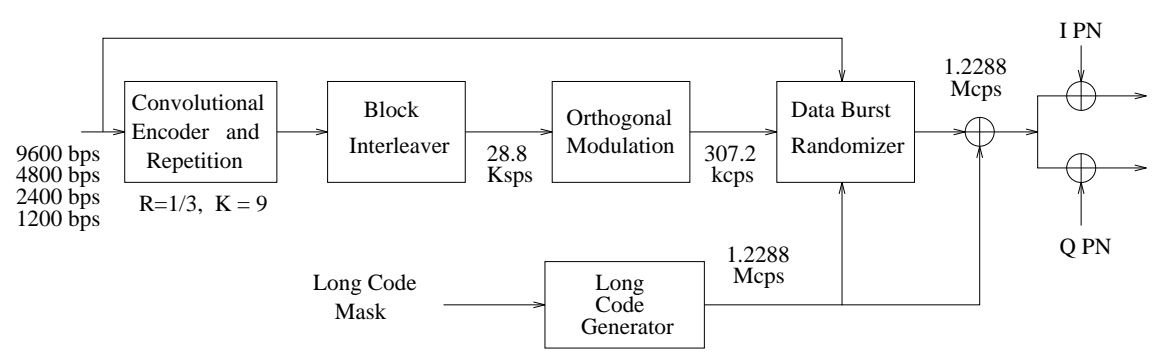

Fig. 3. Rate set 1 uplink traffic channel generation

\subsection{Information Redundancy on the Downlink Traffic Channel}

On the downlink traffic channel, information bits from the vocoder are coded by a convolution encoder as shown in Figure 4. The convolutional encoder has 1-bit input, 2-bit output, and 8-bit memory. Initially the 8 memory bits are filled with all 0s. For every bit of input, the convolutional encoder outputs 2 bits. In coding theory, each bit of output is called a code symbol. Such a convolutional encoder is called a half-rate convolutional encoder [1. Because of the 8-bit memory, each code symbol is related to 9 information bits. The number 9 is called the constraint length of the convolutional encoder. The half-rate convolutional encoder with constraint length of 9 is also called a $(2,1,8)$ convolutional encoder.

Let $b=\left(b_{0}, b_{1}, b_{2}, \ldots\right)$ denote the input sequence entering the convolutional encoder. The two output sequences

$$
v^{(1)}=\left(v_{0}^{(1)}, v_{1}^{(1)}, v_{2}^{(1)}, \ldots\right)
$$




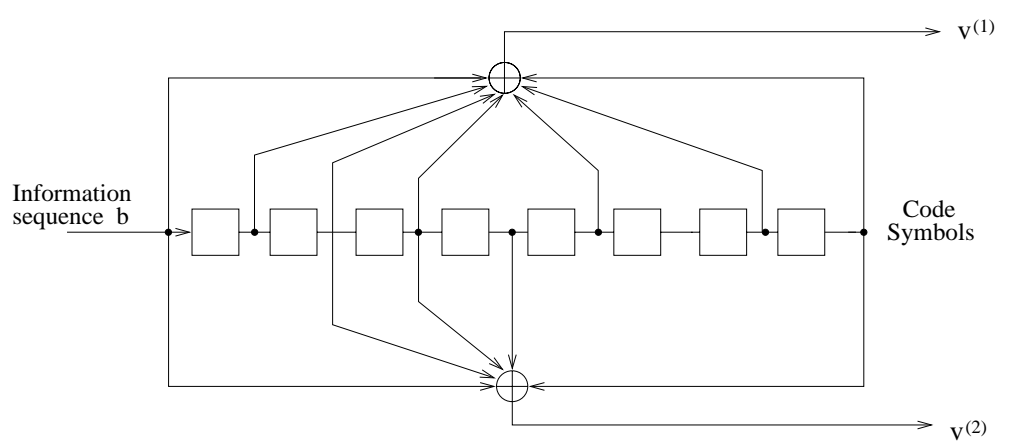

Fig. 4. Half-rate convolutional encoder

and

$$
v^{(2)}=\left(v_{0}^{(2)}, v_{1}^{(2)}, v_{2}^{(2)}, \ldots\right)
$$

can be computed as follows:

$$
\begin{aligned}
& v_{l}^{(1)}=b_{l}+b_{l-1}+b_{l-2}+b_{l-3}+b_{l-5}+b_{l-7}+b_{l-8}, \\
& v_{l}^{(2)}=b_{l}+b_{l-2}+b_{l-3}+b_{l-4}+b_{l-8} .
\end{aligned}
$$

where $b_{l-i}=0$ for all $l<i$. The two output sequences are multiplexed into a single sequence, called the code word. Let $v=\left(v_{0}^{(1)}, v_{0}^{(2)}, v_{1}^{(1)}, v_{1}^{(2)}, v_{2}^{(1)}, v_{2}^{(2)}, \ldots\right)$ denote the code word. From (6) and (7), the code word satisfies the following equation:

$$
v H=0,
$$

where $H$ is a semi-infinite matrix given by

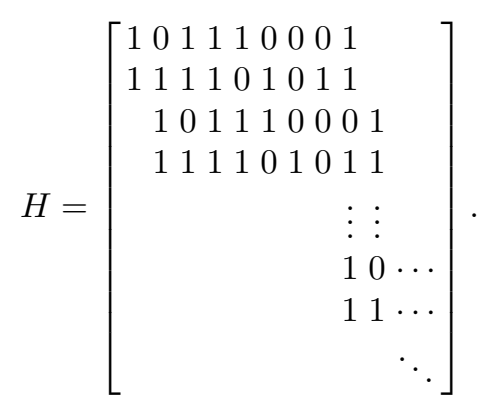

In equation (8), the code word is treated as a semi-infinite sequence since the input sequence to the convolutional encoder may be semi-infinite. When the input sequence to the convolutional encoder is truncated to $k$ bits and the last 8 bits are all zero, the code symbol sequence has length $n=2 k$ and satisfies the following equation:

$$
v[H]_{n}=0,
$$

where $[H]_{n}$ is composed of the first $n$ rows and $n / 2+8$ columns of $H$. 
Recall that the information bits entering the convolutional encoder are structured in 20-ms frames and the last 8 bits of each frame are set to zero. As a consequence, the code symbols in every 20-ms frame satisfy equation (9). Thus, every frame on the downlink traffic channel contains redundant information. The redundant information can be used to solve for the long code sequence.

Except the convolutional encoding, symbol repetition also incurs redundant information. The code symbols from the convolutional encoder are repeated whenever the information rate is lower than $9.6 \mathrm{kbps}$. Every code symbol at the $4.8 \mathrm{kbps}$ rate is repeated 1 time, every code symbol at the $2.4 \mathrm{kbps}$ rate is repeated 3 times, and every code symbol at the $1.2 \mathrm{kbps}$ rate is repeated 7 times. Every frame contains 384 code symbols after repetition. Let $u=\left(u_{1}, u_{2}, \cdots\right.$, $\left.u_{384}\right)$ denote the frame after code symbol repetition. If each code symbol appears two times in $u$, it is obvious that

$$
u E_{2}=0,
$$

where $E_{2}$ is a matrix described by

$$
E_{2}=\left[\begin{array}{ccccc}
1 & 0 & 0 & \cdots & 0 \\
1 & 0 & 0 & \cdots & 0 \\
0 & 1 & 0 & & 0 \\
0 & 1 & 0 & & 0 \\
& & \ddots & \\
0 & 0 & 0 & \cdots & 1 \\
0 & 0 & 0 & \cdots & 1
\end{array}\right] .
$$

If we sample every other code symbol in $u=\left(u_{1}, u_{2}, \cdots, u_{384}\right)$, the sampled code symbols $\left(u_{1}, u_{3}, \cdots, u_{383}\right)$ should satisfy equation (9) since $\left(u_{1}, u_{3}, \cdots, u_{383}\right)$ are the output symbols of the convolutional encoder. Mathematically, the sampling of every other symbols of $u=\left(u_{1}, u_{2}, \cdots, u_{384}\right)$ can be described by the matrix multiplication $u D_{2}$, where

$$
D_{2}=\left[\begin{array}{ccccc}
1 & 0 & 0 & \cdots & 0 \\
0 & 0 & 0 & & 0 \\
0 & 1 & 0 & & 0 \\
\vdots & & \ddots & \\
0 & 0 & 0 & & 1 \\
0 & 0 & 0 & \cdots & 0
\end{array}\right] .
$$

Substituting $v$ in (9) by $u D_{2}$, we have

$$
u D_{2}[H]_{192}=0 .
$$

Generally, if each code symbol in $u=\left(u_{1}, u_{2}, \cdots, u_{384}\right)$ appears $k$ times, where $k=1,2,4,8$, then the code symbol frame $u=\left(u_{1}, u_{2}, \cdots, u_{384}\right)$ satisfies the following equations:

$$
\begin{aligned}
& u E_{k}=0, \\
& u D_{k}[H]_{384 / k}=0,
\end{aligned}
$$


where $E_{1}=0, D_{1}=\mathbf{I}, E_{4}, D_{4}, E_{8}$, and $D_{8}$ can be derived in similar ways as $E_{2}$ and $D_{2}$.

\subsection{Recovery of the Long Code Sequence}

To recover the long code sequence, the eavesdropper intercepts the downlink traffic channel, demodulates the intercepted data frames, and despreads the data frames using the Walsh code specific to the channel. Let $r=\left(r_{1}, r_{2}, \cdots, r_{384}\right)$ denote the data frame after despreading. Correspondingly, let $c=\left(c\left(t_{1}\right), c\left(t_{2}\right), \cdots\right.$, $\left.c\left(t_{384}\right)\right)$ denote the 384 bits of the long code sequence used for scrambling, $s=$ $\left(s_{1}, s_{2}, \cdots, s_{384}\right)$ denote the output of the block interleaver, and $u=\left(u_{1}, u_{2}, \cdots\right.$, $\left.u_{384}\right)$ denote the input to the block interleaver. Figure 5 describes the relationship among these notations on the downlink traffic channel.

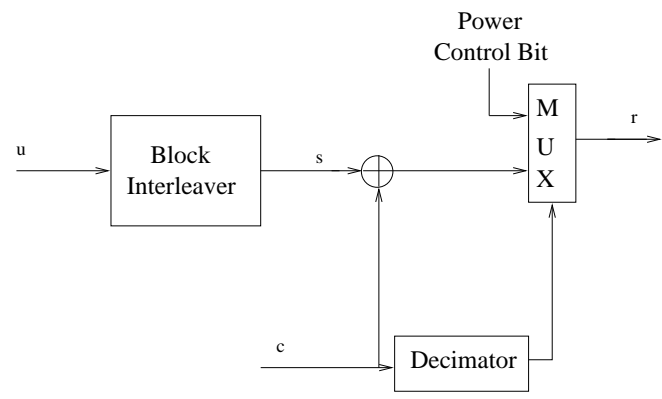

Fig. 5. Block interleaving and scrambling

The block interleaver is a permutation of the 384 input code symbols. The input symbols are entered as a $24 \times 16$ array and the interleaver produces a $24 \times 16$ output array. Table 1 describes the input array. The table is read down by columns from the left to the right. That is, the first input symbol $u_{1}$ is at the top left, the second input symbol $u_{2}$ is just below the first input symbol, and the 25 th input symbol $u_{25}$ is just to the right of the first input symbol. The output array is given by Table 2, which is read the same way as Table 1 , that is, the first output symbol is $u_{1}$, the second output symbol is $u_{65}$, and the 25 th output symbol is $u_{9}$.

Mathematically, the block interleaver can be represented by a permutation matrix $P$, namely,

$$
s=u P,
$$

where $P$ is a $384 \times 384$ matrix, each row and column has only one 1 .

For the moment, let's assume that the power control bits were not transmitted through the downlink traffic channel. Then we have

$$
r=s \oplus c .
$$


Table 1. Downlink traffic channel interleaver input

\begin{tabular}{|c|c|c|c|c|c|c|c|c|c|c|c|c|c|c|c|}
\hline 1 & 25 & 49 & 3 & 97 & 121 & 45 & 169 & 193 & 217 & 241 & 165 & 289 & 313 & 7 & 361 \\
\hline & 26 & 50 & 4 & 98 & & & 0 & 4 & 210 & 242 & & 290 & 14 & 38 & 62 \\
\hline & 27 & 51 & 75 & 99 & 12 & 14 & 17 & 15 & 219 & 24 & 267 & 29 & 315 & $39:$ & 63 \\
\hline & 28 & 52 & 76 & 100 & 12 & 14 & 17 & 196 & 220 & 244 & 268 & 29 & 316 & 40 & 364 \\
\hline & 29 & 53 & 77 & 101 & 125 & 14 & 17 & 197 & 221 & 24 & 269 & 29 & 317 & 341 & 365 \\
\hline & 30 & 54 & 78 & 10 & 126 & 15 & 17 & 198 & 22 & 24 & 270 & 294 & 318 & 342 & 366 \\
\hline & 31 & 55 & 79 & 103 & 27 & 15 & 17 & 199 & 223 & 247 & 27 & 29 & 319 & 343 & 367 \\
\hline & 32 & 56 & 80 & 10 & 12 & & & 0 & 22 & 24 & & & 20 & 34 & 68 \\
\hline & 33 & 57 & 81 & 10 & 129 & 1. & & & 22 & 24 & 2 & 2 & 321 & 345 & 369 \\
\hline 0 & 34 & 58 & 81 & 10 & 130 & 15 & & & 22 & 25 & & & 322 & 346 & 70 \\
\hline 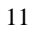 & 35 & 59 & 83 & 10 & 13 & 1. & & 20 & 22 & 25 & 27 & 2 & 323 & 347 & 371 \\
\hline 12 & 36 & 60 & 84 & 10 & 132 & 15 & & 20 & 22 & 25 & & & 324 & 348 & 72 \\
\hline 3 & 37 & 61 & 85 & 1 & 13 & 1. & & 20 & 22 & 25 & 2 & & 325 & 349 & 373 \\
\hline 14 & 38 & 62 & 86 & 1 & 132 & & & & 2 & & & & 326 & 350 & 374 \\
\hline 5 & 39 & 63 & 87 & 11 & 13 & 1. & & 20 & 23 & 2 & 2 & 3 & 327 & 351 & 375 \\
\hline 16 & 40 & 64 & 88 & 1 & 13 & 16 & & 20 & 23 & 250 & 28 & 30 & 328 & 35 & 376 \\
\hline 17 & 41 & 65 & 89 & & 137 & 16 & & 20 & 23 & 257 & 28 & 31 & 329 & 353 & 377 \\
\hline 8 & 42 & 66 & 90 & & & & & & & & & & 330 & 354 & 378 \\
\hline 19 & 43 & 67 & 91 & & 139 & 16 & 18 & 2 & 23 & 259 & 28 & 307 & 331 & 355 & 379 \\
\hline 20 & 44 & 68 & 92 & 11 & & 10 & & & & 260 & 28 & 30 & 332 & 356 & 380 \\
\hline 21 & 45 & 69 & 93 & & 141 & 16 & & 21 & 23 & 261 & 28 & 309 & 33 & 357 & 381 \\
\hline & 46 & 70 & 94 & & 14 & 16 & & 21 & 23 & 262 & 286 & 310 & 34 & 358 & 382 \\
\hline 3 & 47 & 71 & 95 & & 143 & 16 & & 21 & 23 & 263 & 287 & & 35 & 359 & 383 \\
\hline 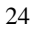 & 48 & 72 & 96 & 120 & 144 & 168 & 192 & 216 & 240 & 264 & 288 & 312 & 336 & 360 & 384 \\
\hline
\end{tabular}

Table 2. Downlink traffic channel interleaver output

\begin{tabular}{|c|c|c|c|c|c|c|c|c|c|c|c|c|c|c|c|}
\hline 1 & 9 & 5 & 13 & 3 & 11 & 7 & 15 & 2 & 10 & 6 & 14 & 4 & 12 & 8 & 16 \\
\hline 65 & 73 & 69 & 77 & 67 & 75 & 71 & 79 & 66 & 74 & 70 & 78 & 68 & 76 & 72 & 80 \\
\hline 129 & 137 & 133 & 141 & 131 & 139 & 135 & 143 & 130 & 138 & 134 & 142 & 132 & 140 & 136 & 144 \\
\hline 193 & 201 & 197 & 205 & 195 & 203 & 199 & 207 & 194 & 202 & 198 & 206 & 196 & 204 & 200 & 208 \\
\hline 257 & 265 & 261 & 269 & 259 & 267 & 263 & 271 & 258 & 266 & 262 & 270 & 260 & 268 & 264 & 272 \\
\hline 321 & 329 & 325 & 333 & 323 & 331 & 327 & 335 & 322 & 330 & 326 & 334 & 324 & 332 & 328 & 336 \\
\hline 33 & 41 & 37 & 45 & 35 & 43 & 39 & 47 & 34 & 42 & 38 & 46 & 36 & 44 & 40 & 48 \\
\hline 97 & 105 & 101 & 109 & 99 & 107 & 103 & 111 & 98 & 106 & 102 & 110 & 100 & 108 & 104 & 112 \\
\hline 161 & 169 & 165 & 173 & 163 & 171 & 167 & 175 & 162 & 170 & 166 & 174 & 164 & 172 & 168 & 176 \\
\hline 225 & 233 & 229 & 237 & 227 & 235 & 231 & 239 & 226 & 234 & 230 & 238 & 228 & 236 & 232 & 240 \\
\hline 289 & 297 & 293 & 301 & 291 & 299 & 295 & 303 & 290 & 298 & 294 & 302 & 292 & 300 & 296 & 304 \\
\hline 353 & 361 & 357 & 365 & 355 & 363 & 359 & 367 & 354 & 362 & 358 & 366 & 356 & 364 & 360 & 368 \\
\hline 17 & 25 & 21 & 29 & 19 & 27 & 23 & 31 & 18 & 26 & 22 & 30 & 20 & 28 & 24 & 32 \\
\hline 81 & 89 & 85 & 93 & 83 & 91 & 87 & 95 & 82 & 90 & 86 & 94 & 84 & 92 & 88 & 96 \\
\hline 145 & 153 & 149 & 157 & 147 & 155 & 151 & 159 & 146 & 154 & 150 & 158 & 148 & 156 & 152 & 160 \\
\hline 209 & 217 & 213 & 221 & 211 & 219 & 215 & 223 & 210 & 218 & 214 & 222 & 212 & 220 & 216 & 224 \\
\hline 273 & 281 & 277 & 285 & 275 & 283 & 279 & 287 & 274 & 282 & 278 & 286 & 276 & 284 & 280 & 288 \\
\hline 337 & 345 & 341 & 349 & 339 & 347 & 343 & 351 & 338 & 346 & 342 & 350 & 340 & 348 & 344 & 352 \\
\hline 49 & 57 & 53 & 61 & 51 & 59 & 55 & 63 & 50 & 58 & 54 & 62 & 52 & 60 & 56 & 64 \\
\hline 113 & 121 & 117 & 125 & 115 & 123 & 119 & 127 & 114 & 122 & 118 & 126 & 116 & 124 & 120 & 128 \\
\hline 177 & 185 & 181 & 189 & 179 & 187 & 183 & 191 & 178 & 186 & 182 & 190 & 180 & 188 & 184 & 192 \\
\hline 241 & 249 & 245 & 253 & 243 & 251 & 247 & 255 & 242 & 250 & 246 & 254 & 244 & 252 & 248 & 256 \\
\hline 305 & 313 & 309 & 317 & 307 & 315 & 311 & 319 & 306 & 314 & 310 & 318 & 308 & 316 & 312 & 320 \\
\hline 369 & 377 & 373 & 381 & 371 & 379 & 375 & 383 & 370 & 378 & 374 & 382 & 372 & 380 & 376 & 384 \\
\hline
\end{tabular}


From (14) and (15),

$$
u=(r \oplus c) P^{-1} .
$$

Substituting (16) into (12) and (13),

$$
\begin{aligned}
& (r \oplus c) P^{-1} E_{k}=0, \\
& (r \oplus c) P^{-1} D_{k}[H]_{384 / k}=0,
\end{aligned}
$$

where $k$ is equal to the number of times each code symbol appears in the frame.

Let $C\left(t_{0}\right)=\left(c\left(t_{0}\right), c\left(t_{0}-1\right), \cdots, c\left(t_{0}-41\right)\right)$ denote the state of the LFSR that the eavesdropper would like to solve for. By (5), $c=\left(c\left(t_{1}\right), c\left(t_{2}\right), \cdots, c\left(t_{384}\right)\right)$ can be represented as

$$
c=C\left(t_{0}\right) \hat{A} .
$$

where $\hat{A}=\left(A^{t_{1}-t_{0}-1}, A^{t_{2}-t_{0}-1}, \ldots, A^{t_{384}-t_{0}-1}\right) a$.

Substituting (19) into (17) and (18), we have the following equations with $c\left(t_{0}\right), c\left(t_{0}-1\right), \cdots, c\left(t_{0}-41\right)$ as variables,

$$
\begin{aligned}
& \left(r \oplus C\left(t_{0}\right) \hat{A}\right) P^{-1} E_{k}=0, \\
& \left(r \oplus C\left(t_{0}\right) \hat{A}\right) P^{-1} D_{k}[H]_{384 / k}=0 .
\end{aligned}
$$

Corresponding to every $k, k=1,2,4,8$, there are 200 or more linear equations involved in (20) and (21). Solving these linear equations, the state of the LFSR can be recovered.

Now, taking into account of the power control bits, many linear equations in (20) and (21) will no longer hold because of the corruption of the power control bits. The power control subchannel transmits at a rate of one bit every $1.25 \mathrm{~ms}$. Using the puncturing technique, each power control bit which is two symbols long replaces two consecutive downlink traffic channel code symbols. Since the code symbols have a rate of $19.2 \mathrm{ksps}$, there will be one power control bit transmitted within every 24 code symbols. There are 16 possible starting positions for the power control bit. Each position corresponds to one of the first 16 code symbols within a $1.25 \mathrm{~ms}$ period. In each $1.25 \mathrm{~ms}$ period, a total of 24 bits from the long code sequence are used for scrambling. These bits are numbered 0 through 23 . The 4-bit binary number with values 0 through 15 , formed from scrambling bits 23,22,21, and 20, are used to determine the position of the power control bit. Hence, within every 24 code symbols, the last 7 code symbols are not affected by the power control bits. These uncorrupted code symbols include $r_{18+24 i}, r_{19+24 i}, \ldots, r_{24+24 i}, i=0,1, \cdots, 15$. Table 2 outlines the interleaver output uncorrupted by the power control bits. Those symbols in the dotted boxes in Table 2 can be recovered reliably from $r$ and $c$. Correspondingly, those symbols in the dotted boxes in Table 1 can be recovered reliably from $r$ and c. Code symbols in the dotted boxes in Table 1 can be divided into 7 groups. Each group contains 16 consecutive code symbols. The 7 groups are listed as follows: 


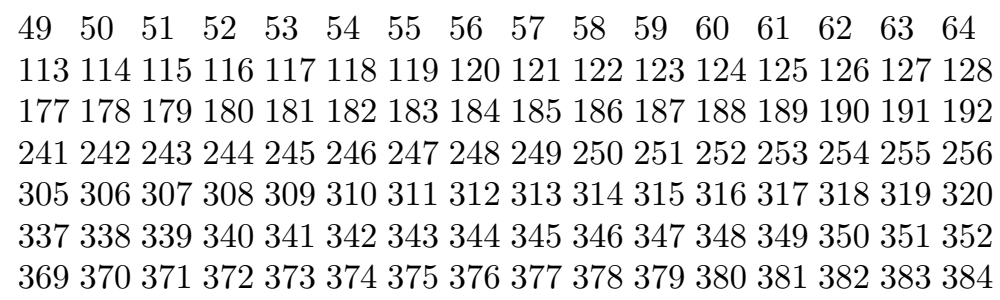

In the following, we will extract those linear equations in (20) and (21) that are only related to the uncorrupted code symbols in $r$, such linear equations are called reliable linear equations.

(1) When the data rate is $19.2 \mathrm{ksps}$, we can not obtain any linear equations from (20) since code symbols are not repeated. In (21), there are 8 reliable linear equations. These reliable linear equations are related with code symbols in the 7 th group, that is, $s_{369}$ through $s_{384}$. Since the constraint length of the $(2,1,8)$ convolutional encoder is 9 , except for the first and the last 8 linear equations, other linear equations in $(21)$ are related to 18 code symbols. From the structure of $H$, it is easy to find that the first 8 linear equations are related to the first $2,4,6,8,10,12,14$, and 16 code symbols respectively. Similarly, the last 8 linear equations are related to the last $2,4,6,8,10,12,14$, and 16 code symbols respectively.

(2) When the data rate is $9.6 \mathrm{ksps}$, there are 56 reliable linear equations in (20), which are related to the code symbols in the 7 groups. There are 4 reliable linear equations in (21), which are related to the code symbols in the 7 th group. Hence, there are a total of 60 reliable linear equations in (20) and (21).

(3) When the data rate is $4.8 \mathrm{ksps}$, there are 86 reliable linear equations in (20) and 2 reliable linear equations in (21), with a total of 86 reliable linear equations in $(20)$ and $(21)$.

(4) When the data rate is $2.4 \mathrm{ksps}$, there are 98 reliable linear equations in (20) and 1 reliable linear equations in (21), with a total of 99 reliable linear equations in $(20)$ and (21).

Hence, when the data rate is not $19.2 \mathrm{ksps}$, an eavesdropper can derive 60 or more reliable linear equations from one data frame. The eavesdropper can solve the reliable linear equations corresponding to the three data rates, 9.6 ksps, $4.8 \mathrm{ksps}$, and $2.4 \mathrm{ksps}$. If there are no solutions corresponding to any of the three data rates. The eavesdropper determines that the data rate should be $19.2 \mathrm{ksps}$. If there is a solution corresponding to one of the three data dates. The long code sequence can be computed and the positions of the power control bits can be determined. Also the corruption caused by the power control bits can be removed by the convolution code. The intercepted code symbols are tested against equations (17) and (18). If the test fails, the solution is incorrect. If there is only one solution passing the test, then long code sequence is found out. If more than one solution pass the tests, then use another frame to test every solution, this process continues until a unique solution is found out. 


\subsection{A Robust Attack}

In the above attack, the eavesdropper needs to solve 4 sets of linear equations corresponding to the 4 data rates, $19.2 \mathrm{ksps}, 9.6 \mathrm{ksps}, 4.8 \mathrm{ksps}$, and $2.4 \mathrm{ksps}$. In the situation when more than one set of linear equations have solutions, the eavesdropper has to determine which solution is correct. This process can be avoided if the eavesdropper can construct a set of linear equations that hold for the 4 data rates. In the following, we will investigate reliable linear equations in (20) and (21) that hold for the 4 data rates.

When the data rate is not $19.2 \mathrm{ksps}$, the code symbols in $u$ are repeated at least one time. As a result $u_{2 i}$ and $u_{2 i+1}, 0 \leq i \leq 192$, must be equal, i.e.,

$$
u_{2 i} \oplus u_{2 i+1}=0, \quad 0 \leq i \leq 192 .
$$

When the data rate is $19.2 \mathrm{ksps}$, the code symbols in $u$ are not repeated. But, from (13), we can get the following equation

$$
u_{383} \oplus u_{384}=0 .
$$

By (22), it can be concluded that (23) holds for the 4 data rates. From Table 2,

$$
\begin{aligned}
& u_{383}=s_{192}, \\
& u_{384}=s_{384} .
\end{aligned}
$$

Since $r_{192}$ and $r_{384}$ are not corrupted by the power control bits, by (15),

$$
\begin{aligned}
& u_{383}=r_{192} \oplus c\left(t_{192}\right), \\
& u_{384}=r_{384} \oplus c\left(t_{384}\right) .
\end{aligned}
$$

Hence, it follows that

$$
c\left(t_{192}\right) \oplus c\left(t_{384}\right)=r_{192} \oplus r_{384} .
$$

Therefore, from every intercepted frame, the eavesdropper can always construct a reliable linear equation which is independent of the date rate associated with the frame. With 42 intercepted frames, the eavesdropper can construct a set of 42 reliable linear equations to solve for the long code sequence. Each frame lasts for $20 \mathrm{~ms}, 42$ frames last for $840 \mathrm{~ms}$, which is less than 1 second. Hence, by eavesdropping the downlink traffic channel 1 second, the eavesdropper can get enough information to recover the long code sequence. After recovering the long code sequence, the eavesdropper can despread the uplink traffic channel.

\section{Concluding Remarks}

The analysis of this paper demonstrates that IS-95 CDMA provides a lowwer than expected level of voice privacy. By eavesdropping the transmission on the downlink traffic channel for one second, an eavesdropper can recover the long 
code sequence used for voice privacy. The vulnerability of the voice privacy may have effect on the security of the authentication process since the long code mask is generated during the authentication process. As details of the authentication protocol are not publicly available due to export restriction, we will leave this problem for future research.

There are several reasons for the weak voice privacy. First, channel coding and symbol repetition leak information about the long code sequence, which is demonstrated by (12) and (13). Channel coding provides error correction capability by introducing redundancy in the transmitted information, while ciphers remove redundancy in the information. When error-correcting codes and ciphers are used in the same channel, the effects of error-correcting codes on ciphers should be carefully examined. Second, the block interleaver does not distribute power control bits in a frame uniformly. From Table 1, there are many consecutive code symbols which are not affected by the power control bits. Third, most importantly, the long code generator is not a good cipher. From (3), the long code sequence is a linear feedback shift register sequence. Linear feedback shift register sequence may be good for spread spectrum purpose but not good for cryptographic purpose. It had better design the two sequences separately, one used for spreading, and the other for voice privacy. It is difficult to design a sequence for both spread spectrum and voice privacy. There are different requirements for the two applications. Hence, a new cipher that provides strong voice privacy is required.

Last, we would like to emphasis that attacks described in this paper have not gone through any field test and we have no intention of performing any test in the future. To achieve a high level of voice privacy, the Telecommunication Industry Association (TIA) TR-45 has created a process for developing enhanced encryption algorithms for the next generation CDMA systems. The enhancement will use 128-bit private keys. Several algorithms, including an algorithm designed by the authors of the paper, have been submitted to TR-45 Ad Hoc Authentication Group (AHAG) for adoption of encryption standard for the next generation CDMA systems.

\section{References}

1. Ajay Dholakia, Introduction to Convolution Codes and Applications, Kluwer Academic Publishers, 1994.

2. Rajian Kuruppillai, Mahi Dontamsetti, Fil Cosentino, Wireless PCS, McGraw-Hill, 1997.

3. TIA/EIA/IS-95-A, Mobile Station-Base Station Compatibility Standard for DualMode Wideband Spread Spectrum Cellular System. 